\title{
Titanium Surface Modification with Carbon Nanotubes. Towards Improved Biocompatibility
}

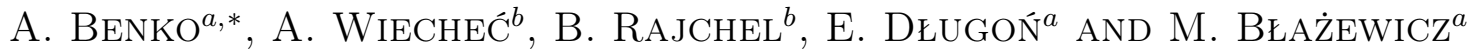 \\ ${ }^{a}$ AGH University of Science and Technology, Faculty of Materials Science and Ceramics, \\ al. A. Mickiewicza 30, 30-059 Kraków, Poland \\ ${ }^{b}$ Institute of Nuclear Physics, Polish Academy of Sciences, Kraków, Poland
}

\begin{abstract}
Subject of this study is surface modification of titanium with thin layers of carbon nanotubes, obtained via an electrophoretic deposition, as a means to improve metal's biocompatibility and provide a suitable matrix for very facile further modifications, if needed. Article presents a preliminary evaluation of the material, using goniometer, scanning electron microscopy and the Raman spectroscopy. The layer is found to be composed of randomly distributed, strongly adhered carbon nanotubes, introducing nanotopography to the surface of titanium. Biological studies were conducted with the human osteoblast-like cell line MG63. Biocompatibility of materials was evaluated using: (a) lactate dehydrogenase cytotoxicity test $(\mathrm{LDH})$ and (b) $\gamma$-H2AX genotoxicity test (presence of DNA double strand breaks). Results confirmed non-toxic character of the tested materials. Moreover, carbon nanotubes layers enhanced the biocompatibility properties of titanium substrate - material with carbon nanotubes possessed lower cellular toxic properties even than pure titanium. The result of this preliminary study are very promising and may serve as a starting point for further studies, including further chemical or biological modification of the obtained materials.
\end{abstract}

DOI: 10.12693/APhysPolA.129.176

PACS: 68.37.Hk, 78.30.Na, 81.15.Pq, 82.45.Mp, 87.85.J-, 87.85.Rs

\section{Introduction}

Titanium is one of the most often applied materials in the stomatology and orthopaedics, due to its high biocompatibility, low density, sufficient strength and yield to be applied in reconstruction of hard tissues and high corrosion resistance [1]. However, titanium also has some flaws: upon being implanted, corrosion rate can be significantly increased under the influence of stress and wear and the surface of titanium and its alloys does not induce spontaneous adhesion of human bone tissue. As a result, a thin biological layer, separating titanium from the bone can be found surrounding the implant [2]. There are many strategies to improve titanium osseointegration and corrosion resistance, most often involving surface chemical modification [3].

In this study we proposed modification of the titanium surface with multiwalled carbon nanotubes (MWCNTs) via the electrophoretic deposition (EPD), which is facile, cost effective, and easily steerable. Meanwhile, MWCNTs where chosen because of their biomimetic to collagen strains structure [4], reported ability to induce bone's regeneration, growth and calcification [5] and suggested antibacterial properties [6]. Moreover, since the CNTs can be easily modified with other bioactive molecules, drugs, etc., we believe that the CNTs layer can provide a suitable platform for any further modifications, as needed. Thus, materials tailored specifically for the current therapeutic need of a specific patient, can be obtained.

*corresponding author; e-mail: akbenko@gmail.com
The aim of the study was to test the potential applicability of the EPD-deposited CNTs layers to improve titanium biocompatibility.

\section{Materials and methods}

Short, multi-walled carbon nanotubes were obtained from the NanoAmor, Inc (Stock\#: 1213NMGS). Vendordefined characteristics of the material can be found at the producer's site. The CNTs were oxidized in the mixture of concentrated acids, yielding tubes that are highly functionalized with polar groups, as described in our previous study [7]. Thus, easily dispersible CNTs with electrical charge were obtained. $35 \mathrm{mg}$ of the CNTs were dispersed in $12 \mathrm{ml}$ of mixture of acetone, ethanol and water $\left(\mathrm{CNTs}_{\mathrm{S}}\right.$ ea) and used during the EPD process. Titanium plate was cut into $10 \times 10 \mathrm{~mm}^{2}$ squares and used as a positive electrode in the EPD method. The deposition was carried out for $30 \mathrm{~s}$, with applied voltage of $30 \mathrm{~V}$, using a previously described setup [7]. Accordingly, Ti_CNTs_ea samples were fabricated.

Wettability of the materials was evaluated using a standard contact angle goniometer (DSA 10 Kruss goniometer). Morphology was studied under a scanning electron microscope (Nova NanoSEM 200, FEI Europe Company), using a Helix detector. Structure of the deposited tubes was evaluated via the Raman microspectroscopy (Thermo Nicolet Almega XR, laser wavelength: $532 \mathrm{~nm}$, objective magnification $100 \times$, pinhole aperture $25 \mu \mathrm{m}$ and spectral resolution of approximately $8 \mathrm{~cm}^{-1}$ ).

In the biocompatibility study, human osteoblast-like cells (cell line MG63; European Collection of Cell Cultures, Salisbury, UK) were seeded on: (a) polystyrene cell culture dishes (PS) (Sarstedt, Germany), (b) titanium plate, (c) Ti_CNTs_ea sample. The cell culture 
was conducted for up to 3 days in conditions described previously in [8]. Cellular cytotoxicity determination was done by measuring the activity of $\mathrm{LDH}$ released into the media from damaged cells using LDH cytotoxicity detection kit, ClonTech. The immunostaining of $\gamma$-H2AX foci was applied for evaluation of genotoxicity basing on presence of the DNA double strand breaks. The one way analysis of variance (ANOVA) and $t$-Student test were performed. The $p$ values equal to or less than 0.05 were considered significant.

\section{Results and discussion}

When compared to titanium, the Ti CNTs ea sample revealed increased wettability, manifested by the decrease in the contact angle value - from $76 \pm 4^{\circ}$ to $30 \pm 2^{\circ}$. This result may be attributed both to increased topography and introduction of polar functional groups during the deposition. Morphology of the layer is presented in Fig. 1. It can be found that the deposit is formed

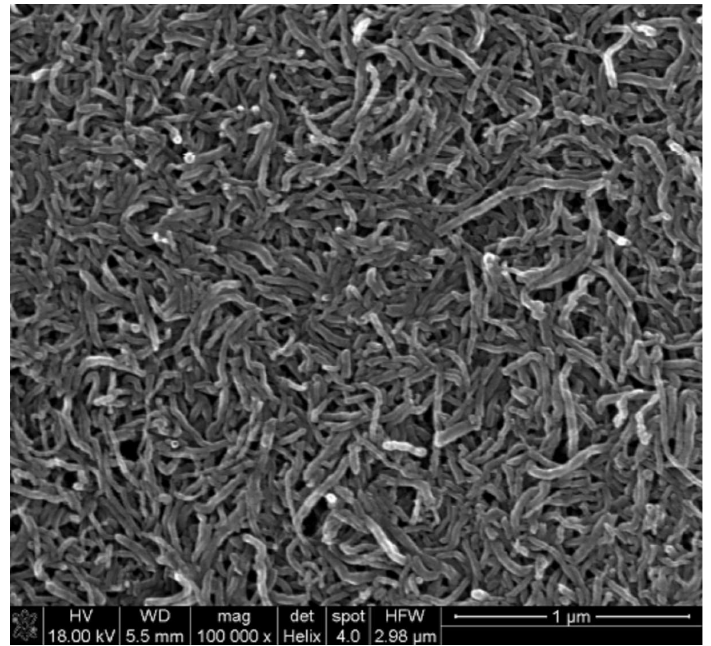

Fig. 1. SEM evaluation of the Ti CNTs ea sample, obtained in high vacuum conditions, with accelerating voltage of $18 \mathrm{kV}$.

of CNTs that are densely packed, and vertically adhered to one another. The tubes are randomly oriented - no distinguishable patterns are observed, indicating that the EPD does not favor deposition in any dimension. The deposit is homogeneous with no flaking or agglomerates visible throughout the layer. This indicates that the CNTs were well dispersed in the solvent, allowing for optimal packing and uniform residual stress distribution within the layer. When it comes to the Raman spectroscopy, the most informative spectral region for graphene based structures is from $1000 \mathrm{~cm}^{-1}$ to $1800 \mathrm{~cm}^{-1}$ and this range is presented in Fig. 2 for the Ti_CNTs_ea sample. In this region, a characteristic for carbon nanotubes, multi-peak tangential mode is present ( $G$ band at approximately $1587 \mathrm{~cm}^{-1}$ ), accompanied by a disorder-induced D1-band (approximately $1352 \mathrm{~cm}^{-1}$ ). To find some of the distinguishable components (such as the D2 band, which is typical of defective graphite like materials),

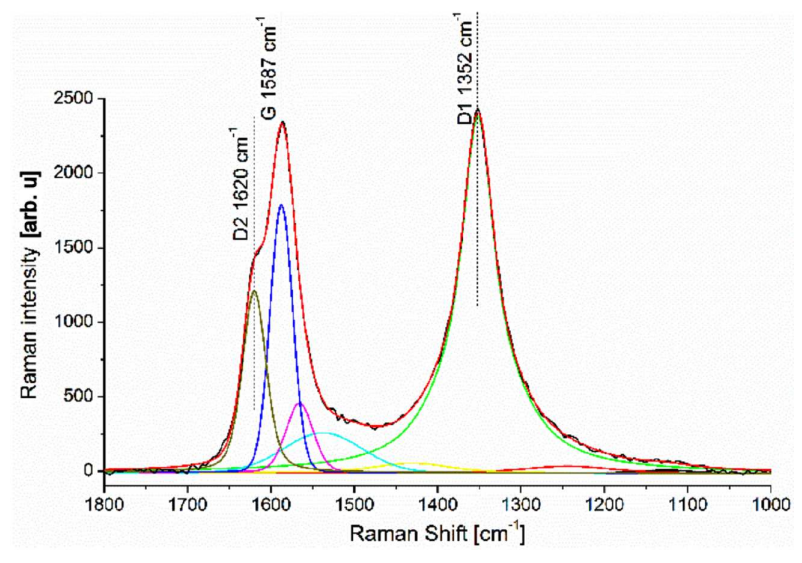

Fig. 2. Raman spectra of the Ti CNTs ea sample using excitation line of $532 \mathrm{~nm}$, region $180 \overline{0}-1100 \mathrm{~cm}^{-1}$.

curve fitting procedure, using Gaussian/Lorentzian shape line was performed. High intensity of the $D 1$ band, together with a presence of a distinguishable $D 2$ band indicate high amount of defects, attributed mostly to the presence of heteroatoms within the functional groups, such as hydroxyl, carboxyl, carbonyl or C-O [7, 9, 10]. Meanwhile, an upshift of the $G$ band from the values found in the literature can be connected to the intercalation of the tubes with the acceptor dopant [11].

\section{TABLE I}

Cytotoxicity of samples measured with LDH assay in first and third day of culturing. Results presented as Means [absorbance $\left.\left(A_{492 \mathrm{~nm}}-A_{690 \mathrm{~nm}}\right)\right] \pm$ standard deviation.

\begin{tabular}{c|c|c|c|c}
\hline \hline & PS & Ti & Ti_CNTs_ea & ANOVA \\
\hline 1st day & $6.3 \pm 0.3$ & $11.0 \pm 1.3$ & $7.4 \pm 0.9$ & $\mathrm{~ns}$ \\
3rd day & $7.8 \pm 0.3$ & $11.6 \pm 0.7$ & $8.9 \pm 0.2$ & $\mathrm{~ns}$ \\
ANOVA & $\mathrm{ns}$ & $\mathrm{ns}$ & $\mathrm{ns}$ &
\end{tabular}

TABLE II

Genotoxicity of samples measured as a fluorescence of $\gamma$-H2AX. Results presented as Mean [arbitrary units] \pm standard deviation.

\begin{tabular}{c|c|c|c|c}
\hline \hline & PS & Ti & Ti_CNTs_ea & ANOVA \\
\hline 1st day & $17.75 \pm 0.08$ & $35.10 \pm 2.84$ & $16.81 \pm 3.81$ & $\mathbf{0 . 0 0 1}$ \\
3rd day & $16.19 \pm 3.33$ & $46.76 \pm 3.23$ & $9.94 \pm 1.61$ & $\mathbf{0 . 0 0 1}$ \\
ANOVA & ns & $\mathbf{0 . 0 1}$ & ns &
\end{tabular}

Cytotoxicity (Table I) and genotoxicity (Table II) detected for titanium modified with carbon nanotubes was similar to the cytotoxicity of culture plates (PS). Statistically insignificant higher level of cytotoxicity was observed for the titanium plate (Table I). However, genotoxicity of titanium was significantly higher when compared to PS and Ti_CNTs_ea samples and was found to increase with time of incubation (up to 3rd day of culture). This indicates that the CNTs layer does not only provide favorable conditions for cells adhesion and growth but also reduces the genotoxicity of titanium increasing its biocompatibility. 


\section{Conclusions}

In the presented study, covering the titanium with highly functionalized MWCNTs resulted in decreased cytotoxicity and genotoxicity of the material in contact with MG63 cells. High level of structural disorder, connected to the presence of significant amount of functional groups, together with an increased surface area of the sample provided a more suitable surface for cells adhesion and growth, when compared to pure titanium. The result of this preliminary study are very promising and may serve as a starting point for future studies, including further chemical or biological modification of the obtained materials.

\section{Acknowledgments}

This work has been supported by National Science Centre (NCN) grants Nos.: 2011/01/B/ST5/06424 and UMO-2013/11/N/ST8/01357

\section{References}

[1] M. Geetha, A.K. Singh, R. Asokamani, A.K. Gogia, Prog. Mater. Sci. 54, 397 (2009).

[2] B. Ratner, A Perspective on Titanium Biocompatibility, Titanium in Medicine, Springer, Berlin 2001, p. 1.
[3] X. Liu, P.K. Chu, C. Ding, Mater. Sci. Eng. R 47, 49 (2004).

[4] J. Beuvelot, C. Bergeret, R. Mallet, V. Fernandez, J. Cousseau, M.F. Basle, D. Chappard, Acta Biomater. 6, 4110 (2010).

[5] P. Newman, A. Minett, R. Ellis-Behnke, H. Zreiqat, Nanomedicine 9, 1139 (2013).

[6] S. Kang, M. Herzberg, D.F. Rodrigues, M. Elimelech, Langmuir 24, 6409 (2008).

[7] A. Przekora, A. Benko, M. Nocun, J. Wyrwa, M. Blazewicz, G. Ginalska, Mater. Sci. Eng. C Mater. Biol. Appl. 45, 287 (2014).

[8] A. Wiechec, E. Stodolak-Zych, A. Fraczek-Szczypta, M. Blazewicz, W.M. Kwiatek, Acta Phys. Pol. A 121, 546 (2012).

[9] A. Jorio, M.A. Pimenta, A.G. Souza, R. Saito, G. Dresselhaus, M.S. Dresselhaus, New J. Phys. 5, 139.131 (2003)

[10] O. Beyssac, B. Goffe, J.P. Petitet, E. Froigneux, M. Moreau, J.N. Rouzaud, Spectrochim. Acta A Mol. Biomol. Spectrosc. 59, 2267 (2003).

[11] S. Costa, E. Borowiak-Palen, Acta Phys. Pol. A 116, $32(2009)$. 